\title{
The endpoint Fefferman-Stein inequality for the strong max- imal function with respect to nondoubling measure
}

Wei Ding

School of Sciences, Nantong University, Nantong 226007, P. R. China.

Communicated by A. Meskhi

\section{Abstract}

Let $d \mu\left(x_{1}, \ldots, x_{n}\right)=d \mu_{1}\left(x_{1}\right) \cdots d \mu_{n}\left(x_{n}\right)$ be a product measure which is not necessarily doubling in $\mathbb{R}^{n}$ (only assuming $\mathrm{d} \mu_{i}$ is doubling on $\mathbb{R}$ for $\left.i=2, \ldots, n\right)$, and $M_{d \mu}^{n}$ be the strong maximal function defined by

$$
M_{d \mu}^{n} f(x)=\sup _{x \in R \in \mathcal{R}} \frac{1}{\mu(R)} \int_{R}|f(y)| d \mu(y),
$$

where $\mathcal{R}$ is the collection of rectangles with sides parallel to the coordinate axes in $\mathbb{R}^{n}$, and $\omega, v$ are two nonnegative functions. We give a sufficient condition on $\omega, \nu$ for which the operator $M_{d \mu}^{n}$ is bounded from $L\left(1+\left(\log ^{+}\right)^{n-1}\right)(\nu d \mu)$ to $L^{1, \infty}(\omega d \mu)$. By interpolation, $M_{d \mu}^{n}$ is bounded from $L^{p}(v d \mu)$ to $L^{p}(\omega d \mu), 1<p<\infty$.

Keywords: Fefferman-Stein inequality, strong maximal function, nondoubling measure, $A^{\infty}$ weights, reverse Hölder's inequality.

2010 MSC: 42B25, 42B37.

(C)2018 All rights reserved.

\section{Introduction}

Since the classical theory of harmonic analysis may be described as centering around the HardyLittlewood maximal operator and its relationship with certain singular integral operators, the maximal function have attracted the attention of a lot of researchers, such as $[1,3-5,8-10,13,16,22,30]$.

Let $\mathcal{B}_{x}$ be a collection of bounded sets containing $x \in \mathbb{R}^{n}$, and $v$ be a positive measure. Given a locally integrable function $f$, denote

$$
\mathcal{M} f(x)=\sup _{R \in \mathcal{B}_{x}} \frac{1}{v(R)} \int_{R}|f(y)| d v(y) .
$$

If $\mathcal{B}_{x}$ is the collection of all the cubes containing $x \in \mathbb{R}^{n}$ and whose sides parallel to the coordinate axes, $d v(x)=d x$, then we obtain the usual Hardy-Littlewood maximal function $M f(x)$. When $\mathcal{B}_{x}$ denotes the

Email address: dingwei@ntu.edu.cn (Wei Ding)

doi: $10.22436 /$ jnsa.011.11.07

Received: 2017-12-17 Revised: 2018-07-21 Accepted: 2018-08-15 
collection of all rectangles $R$ containing $x \in \mathbb{R}^{n}$ whose sides parallel to the coordinate axes, $\mathcal{M} \triangleq M_{d v}^{n}$ is the strong maximal operator with respect to measure $d v$. If $d v(x)=d x$, denote $M^{n}=M_{d v}^{n}$.

For every non-negative, locally integrable weight $\omega$, Fefferman and Stein in [11] proved the following well known inequality

$$
\int_{\mathbb{R}^{n}}(M f)^{p}(x) \omega(x) d x \lesssim \int_{\mathbb{R}^{n}}\left(|f(x)|^{p} M \omega(x) d x, 1<p<\infty .\right.
$$

Inequalities of this type are important, for example, they can be used to derive the boundedness of vectorvalued maximal operators. More details can be seen in [11-13]. Similar inequalities were also obtained for singular integral operators in [5]. In this situation, $M \omega(x)$ in the right hand was replaced by $M\left(\omega^{r}\right)^{1 / r}$. The above inequality is also true for the strong maximal function $M^{n}$ if $\omega \in A_{\infty}^{n}[19,21]$.

For the usual Hardy-Littlewood maximal function $M f(x)$, the form of the endpoint Fefferman-Stein inequality is the following

$$
\omega\left(\left\{x \in \mathbb{R}^{n}: \operatorname{Mf}(x)>\lambda\right\}\right) \lesssim \frac{1}{\lambda} \int_{\mathbb{R}^{n}}|f(x)| \operatorname{M\omega }(x) \mathrm{d} x, \lambda>0 .
$$

For strong maximal function $M^{n} f(x)$, it is more complicated. When $n=2$, Mitsis in [21] has obtained that

$$
\omega\left(\left\{x: M^{2}(f)(x)>\lambda\right\}\right) \lesssim \int \frac{|f(x)|}{\lambda}\left(1+\log ^{+} \frac{|f(x)|}{\lambda}\right) M^{2} \omega(x) d x, \lambda>0,
$$

if $\omega \in A_{p}^{2}$ for some $1<p<\infty$. Recently, Luque and Parissis in [19] improved this result to any dimension $n \geqslant 2$ provided only $\omega \in A_{\infty}^{n}$. We want to point out that there is no assumption on the weight to establish Fefferman-Stein inequality for the Hardy-Littlewood maximal function.

The classical theory of one-parameter harmonic analysis for maximal functions and singular integrals on $\left(\mathbb{R}^{n} ; \mu\right)$ has been developed under the assumption that the underlying measure $\mu$ satisfies the doubling property, i.e., there exists a constant $C>0$ such that $\mu(B(x ; 2 r)) \leqslant C \mu(B(x ; r))$ for every $x \in \mathbb{R}^{n}$ and $r>0$. However, some recent results $[20,23,25,31]$ show that it is possible to dispense with the doubling condition for most of the classical theory. It is well known that the use of doubling measure has two main advantages. One is that we can work with nested property. Another one is that the faces of the cubes have measure zero. As in the paper [20,25], we will only maintain the last property. If $\mu$ is a nonnegative Radon measure without mass-points, one can choose an orthonormal system in $\mathbb{R}^{n}$ so that any cube $Q$ with sides parallel to the coordinate axes satisfies the property $\mu(\partial Q)=0$ ([20, Theorem 2]). The profit of this property is the continuity of the measure $\mu$ on cubes which can ensure that there is a Calderón-Zygmund decomposition [20, 25]. For the development of multi-parameter harmonic analysis, we refer the readers to the works in $[2,9,14,15,17]$.

Therefore, there is a nature question: can the Fefferman-Stein inequality be established with a general measure $v$ for the strong maximal operator $M_{\mathrm{d} v}^{n}$ ?

Let $\mu=\mu_{1} \times \mu_{2} \times \cdots \times \mu_{n}$ be a product measure, where $\mu_{i}, i=1, \ldots, n$ are all nonnegative Radon measures without mass-points and complete. The assumption that $\mu_{i}$ are complete is just a technical requirement to allow exchange integral order. For a rectangle $R \subseteq \mathbb{R}^{n}$, we mean a rectangle whose sides parallel to the coordinate axes. Under this kind of product measure, in [7], we investigated the $\mathrm{L}^{\mathrm{p}}(\omega \mathrm{d} \mu)$ boundedness of strong maximal functions $M_{\omega \mathrm{d} \mu}^{n}$ and $M_{\mathrm{d} \mu}^{n}$ when $\omega \in A_{\infty}^{n}$ defined by the following.

Definition 1.1. Let $1<p<\infty$ and $p^{\prime}=p /(p-1)$. We say that a weight $\omega$ satisfies the $A_{p}^{n}(\mu)$ condition if

$$
[\omega]_{A_{\mathcal{p}}^{\mathfrak{n}}(\mu)}=\sup _{R \in \mathcal{R}}\left(\frac{1}{\mu(R)} \int_{R} \omega \mathrm{d} \mu\right)\left(\frac{1}{\mu(R)} \int_{R} \omega^{1-p^{\prime}} d \mu\right)^{p-1}<\infty,
$$

where $\mathcal{R}$ is a collection of all rectangles $R$ whose sides parallel to the coordinate axes. 
We say $\omega \in A_{1}^{n}(\mu)$ if there exists a constant $C>0$ such that

$$
M_{\mathrm{d} \mu}^{n} \omega(x) \leqslant C \omega(x)
$$

for almost every $x \in \mathbb{R}^{n}$.

Define $A_{\infty}^{n}(\mu)$ by

$$
A_{\infty}^{n}(\mu)=\bigcup_{1 \leqslant p<\infty} A_{p}^{n}(\mu) .
$$

Notice that $A_{r}^{n}(\mu) \subseteq A_{q}^{n}(\mu)$ when $r \geqslant q$, and if $\omega \in A_{\infty}^{n}(\mu)$, then $\omega \in A_{p}^{n}(\mu)$ for some $1<p<\infty$. It is easy to see that if $\omega \in A_{p}^{n}(\mu)$ for some $1<p<\infty, \omega_{i}\left(x_{i}\right)=\omega\left(x_{1}, \ldots, x_{i-1}, \cdot, x_{i+1}, \ldots, x_{n}\right) \in A_{p}^{1}\left(\mu_{i}\right)$ uniformly with respect to $x_{1}, \ldots, x_{i-1}, x_{i+1}, \ldots, x_{n}$. It has been proved in [7] that the behavior of $A_{\infty}^{n}(\mu)$ and the relationship between $A_{p}^{n}(\mu)$ weights and the strong maximal function $M_{d \mu}^{n}$ are very similar to the classical case when we add some conditions to the product measure $\mu$.

There are also some interesting results about two weights. We refer the reader to the work in $[6,24,26-$ 28]. In [26, 27], Pérez provided a sufficient condition on weights $\omega, v$ to ensure the boundedness of the general maximal functions $M_{\wp}$ including the boundedness of $M_{d x}^{n}$ from $L^{p}(\omega)$ to $L^{q}(v)$. More precisely, if the couple of weights $(\omega, v)$ satisfies the following condition: there are constants $0<\lambda<1,0<\mathrm{c}=$ $c(\lambda)<\infty$ such that for all measurable sets $E$

$$
\omega\left(\left\{x: M_{\wp}(x)>\lambda\right\}\right) \leqslant c \omega(E),
$$

which is weaker than the $A_{\infty}^{n}$ condition, and

$$
\sup _{B} \frac{1}{|B|} \int_{B} \omega(y) d y\left(\sup _{B} \frac{1}{|B|} \int_{B} v(y)^{\left(1-p^{\prime}\right) r} d y\right)^{(p-1) / r}<\infty
$$

for some $1<r<\infty$, then $M_{\wp}$ is bounded from $L^{p}(\omega)$ to $L^{q}(v)$.

This idea can be used to our strong maximal function $M_{d \mu}^{n}$. A couple of weights $(\omega, v)$ is said to be satisfied condition (A), if

$$
\left(\frac{1}{\mu(R)} \int_{R} \omega \mathrm{d} \mu\right) \cdot \sup _{x \in R} v^{-1}(x) \leqslant c
$$

for all rectangles $R$ in $\mathbb{R}^{n}$. Then the main result of the current paper is the following.

Theorem 1.2. Assume that $\mu(x)=\mu_{1}\left(x_{1}\right) \cdot \mu_{2}\left(x_{2}\right) \cdots \mu_{n}\left(x_{n}\right)$ is a product measure where $\mu_{i}, i=1, \ldots, n$ are all nonnegative Radon measures in $\mathbb{R}$ without mass-points and complete. Assume also that each $\mu_{\mathrm{i}}$ for $2 \leqslant i \leqslant n$ is doubling on $\mathbb{R}$. If $(\omega, v)$ is a couple of weights such that $\omega \in A_{\infty}^{n}=A_{\infty}^{n}(\mu)$ and that the condition $(A)$ holds, then

$$
\omega\left(\left\{x: M_{d \mu}^{n}(f)(x)>\lambda\right\}\right) \lesssim \int \frac{|f(x)|}{\lambda}\left(1+\left(\log ^{+} \frac{|f(x)|}{\lambda}\right)^{n-1}\right) v(x) d \mu(x),
$$

where $\omega(E)$ denotes $\int_{E} \omega(x) d \mu(x)$ for every $\mu$-measurable set $E$.

By interpolation, the above endpoint two weights Fefferman-Stein inequality implies the strong two weights Fefferman-Stein inequality.

Theorem 1.3. Assume that $\mu(x)=\mu_{1}\left(x_{1}\right) \cdot \mu_{2}\left(x_{2}\right) \cdots \mu_{n}\left(x_{n}\right)$ is a product measure where $\mu_{i}, i=1, \ldots, n$ are all nonnegative Radon measures in $\mathbb{R}$ without mass-points and complete. Assume also that each $\mu_{\mathrm{i}}$ for $2 \leqslant \mathrm{i} \leqslant \mathrm{n}$ is doubling on $\mathbb{R}$. If $(\omega, v)$ is a couple of weights such that $\omega \in A_{\infty}^{n}$ and that the condition $(A)$ holds, then

$$
\left\|M_{\mathrm{d} \mu}^{\mathrm{n}}(\mathrm{f})\right\|_{\mathrm{L}^{\mathrm{p}}(\omega \mathrm{d} \mu)} \lesssim\left\|M_{\mathrm{d} \mu}^{\mathrm{n}}(\mathrm{f})\right\|_{\mathrm{L}^{\mathrm{p}}(v \mathrm{~d} \mu)}, 1<p<\infty .
$$

It is easy to check that $\left(\omega, M_{d \mu}^{n} \omega\right)$ satisfies condition (A), and then we have. 
Corollary 1.4. Assume that $\mu(x)=\mu_{1}\left(x_{1}\right) \cdot \mu_{2}\left(x_{2}\right) \cdots \mu_{n}\left(x_{n}\right)$ is a product measure where $\mu_{i}, i=1, \ldots, n$ are all nonnegative Radon measures in $\mathbb{R}$ without mass-points and complete. Assume also that each $\mu_{\mathrm{i}}$ for $2 \leqslant \mathrm{i} \leqslant \mathrm{n}$ is doubling on $\mathbb{R}$, and $\omega \in A_{\infty}^{n}$, then

$$
\omega\left(\left\{x: M_{d \mu}^{n}(f)(x)>\lambda\right\}\right) \lesssim \int \frac{|f(x)|}{\lambda}\left(1+\left(\log ^{+} \frac{|f(x)|}{\lambda}\right)^{n-1}\right) M_{d \mu}^{n} \omega(x) d \mu(x),
$$

and

$$
\left\|M_{\mathrm{d} \mu}^{n}(f)\right\|_{L^{p}(\omega \mathrm{d} \mu)} \lesssim\left\|M_{\mathrm{d} \mu}^{n}(f)\right\|_{L^{p}\left(M_{\mathrm{d} \mu}^{n} \omega \mathrm{d} \mu\right)}, 1<p<\infty
$$

Let $\mathrm{d} \mu=\mathrm{dx}$, then the above corollary is the main theorem of [19]. By changing variables, in the above results, the product measure $\mu$ can be assumed that $\mu_{i}, i=1, \ldots, n$ are all nonnegative Radon measures in $\mathbb{R}$ without mass-points and complete, permitted only one direction with non-doubling condition.

The organization of the paper is as follows. Section 2 gives some auxiliary lemmas, such as reverse Hölder's inequality of weights $A_{\infty}^{n}(\mu)$, and the asymptotic estimate of the $L^{p}(d \mu)$ norm of $M_{\omega d \mu}^{n}$ as $p \rightarrow 1^{+}$. In last section, we give the proof of Theorem 1.2.

Finally, we make some conventions. Throughout the paper, $c$ denotes a positive constant that is independent of the main parameters involved, but whose value may vary from line to line. Constants with subscript, such as $c_{1}$, do not change in different occurrences. We denote $f \leqslant c g$ by $f \lesssim g$. If $f \lesssim g \lesssim f$, we write $f \approx g$. In order to indicate the dependence of the constant on some parameter $n$ (say), we write $A \lesssim n$ B.

\section{Auxiliary lemmas}

In this section, firstly we give some lemmas about weights $A_{\infty}^{n}(\mu)$ obtained in [7].

Lemma 2.1. Let $\mu$ be a nonnegative Radon measure. If $\omega \in A_{\infty}^{n}(\mu)$, then for $\forall 0<\alpha<1$, there is a positive constant $\beta<1$ such that whenever $F$ is a measurable set of a rectangle $R$, we have

$$
\frac{\mu(F)}{\mu(R)} \leqslant \alpha \text { implies } \frac{\omega(F)}{\omega(R)} \leqslant \beta,
$$

which is equivalent to say that for $\forall 0<\alpha^{\prime}<1$, there is a positive constant $\beta^{\prime}<1$ such that whenever $\mathrm{F}$ is a measurable set of a rectangle $\mathrm{R}$,

$$
\frac{\mu(F)}{\mu(R)} \geqslant \alpha^{\prime} \text { implies } \frac{\omega(F)}{\omega(R)} \geqslant \beta^{\prime}
$$

Lemma 2.2. Assume that $\mu=\mu_{1} \times \mu_{2} \times \cdots \times \mu_{n}$ is a product measure, where $\mu_{i}, i=1, \ldots, n$ are all nonnegative Radon measures without mass-points and complete. If $\omega \in A_{\infty}^{n}(\mu)$, then $\omega$ satisfies a reverse Hölder's inequality, that is, there exist two positive constants $\mathrm{c}$ and $\delta$ such that for every rectangle $\mathrm{R}$

$$
\left(\frac{1}{\mu(R)} \int_{R} \omega^{1+\delta} d \mu\right)^{1 /(1+\delta)} \leqslant \frac{c}{\mu(R)} \int_{R} \omega d \mu
$$

and $\mathrm{c}$ may be taken as close to 1 as $\delta \rightarrow 0^{+}$.

All the proofs of above lemmas can be seen in [7], and we omit it. If $\omega \in A_{p}^{n}(\mu), p>1$, then $\omega^{1-p^{\prime}} \in A_{p^{\prime}}^{n}(\mu)$, where $1 / p+1 / p^{\prime}=1$. Consequently, by Lemma 2.2 , it is easy to deduce the following result.

Lemma 2.3. Let $p>1$, and $\omega \in A_{p}^{n}(\mu)$, then there is an $\varepsilon>0$ such that $\omega \in A_{p-\varepsilon}^{n}(\mu)$. 
Let $x=\left(x_{1}, \ldots, x_{n}\right)$ and $\mu=\mu_{1} \times \mu_{2} \times \cdots \times \mu_{n}$. For convenience, fixed $x_{n} \in \mathbb{R}$, denote $d \omega^{\prime}=$ $\omega\left(x^{\prime}, x_{n}\right) d \mu^{\prime}, x^{\prime}=\left(x_{1}, \ldots, x_{n-1}\right)$ and $d \mu^{\prime}=d \mu_{1} \cdots d \mu_{n-1}$. For some $0<\epsilon<1$, let $\left\{R_{k}\right\}$ be a sequence of rectangles in $\mathbb{R}^{n}$. $\left\{R_{k}\right\}$ is said to have property $P_{1}$ : if

$$
\mu\left(R_{k} \cap \bigcup_{i<k} R_{i}\right) \leqslant \epsilon \mu\left(R_{k}\right) .
$$

$\left\{R_{k}\right\}$ is said to satisfy property $P_{2}$ : if its side lengths in the $x_{n}$ direction are decreasing and

$$
\mu\left(R_{k} \cap \bigcup_{i<k} \hat{R}_{i}\right) \leqslant \epsilon \mu\left(R_{k}\right),
$$

where $\hat{R}$ is the rectangle with the same center as $R$ and whose sides parallel to the first $n-1$ coordinate axes have the same lengths as the corresponding sides of $R$, and the side of $\hat{R}$ which is parallel to the $n$-th coordinate axis has length equal to three times the length of the corresponding side of $R$.

Lemma 2.4. Assume that $\mu(x)=\mu_{1}\left(x_{1}\right) \mu_{2}\left(x_{2}\right) \cdots \mu_{n}\left(x_{n}\right)$ is a product measure where $\mu_{i}, i=1, \ldots, n$ are all nonnegative Radon measures in $\mathbb{R}$. Let $\left\{\mathrm{R}_{\mathrm{k}}\right\}$ be a sequence of rectangles in $\mathbb{R}^{\mathrm{n}}$ satisfying property $\mathrm{P}_{2}$, and $\mathrm{S}_{\mathrm{k}}^{x_{n}}$ be the slice of $R_{k}$ at $x_{n}$. Then $\left\{S_{k}^{x_{n}}\right\}$ satisfies property $P_{1}$ for any $x_{n}$, that is

$$
\mu^{\prime}\left(S_{k}^{x_{n}} \cap \bigcup_{i<k} S_{i}^{x_{n}}\right) \leqslant \epsilon \mu^{\prime}\left(S_{k}^{x_{n}}\right) .
$$

Proof. Since $R_{k}$ is a rectangle in $\mathbb{R}^{n}$, we may assume $R_{k}=I_{k} \times J_{k}$ where $J_{k}$ is the one-dimensional projection to the $x_{n}$ axes and $I_{k}$ is a rectangle in $\mathbb{R}^{n-1}$. The conclusion is obvious if $x_{n} \notin J_{k}$. When $x_{n} \in J_{k}$, setting $J=\left\{i<k, S_{k}^{x_{n}} \cap S_{i}^{x_{n}} \neq \emptyset\right\}$, one has

$$
R_{k} \cap \bigcup_{i \in J} R_{i} \subseteq R_{k} \cap \bigcup_{i<k} R_{i}
$$

By the assumption that the side lengths of the $x_{n}$ direction are decreasing in $\left\{R_{k}\right\}$, one has

$$
R_{k} \cap \bigcup_{i \in J} \hat{R}_{i}=\left(\bigcup_{i \in J} S_{k}^{x_{n}} \cap S_{i}^{x_{n}}\right) \times J_{k}
$$

Hence by the property $P_{2}$, one has

$$
\mu^{\prime}\left(\bigcup_{i \in J} S_{k}^{x_{n}} \cap S_{i}^{x_{n}}\right) \mu_{n}\left(J_{k}\right)=\mu\left(R_{k} \cap \bigcup_{i \in J} \hat{R}_{i}\right) \leqslant \mu\left(R_{k} \cap \bigcup_{i<k} \hat{R}_{i}\right) \leqslant \epsilon \mu\left(R_{k}\right),
$$

which yields our desired result immediately.

Lemma 2.5. Assume that $\mu(x)=\mu_{1}\left(x_{1}\right) \mu_{2}\left(x_{2}\right) \cdots \mu_{n}\left(x_{n}\right)$ is a product measure where $\mu_{i}, i=1, \ldots, n$ are all nonnegative Radon measures in $\mathbb{R}$ without mass-points and complete. Assume also that each $\mu_{\mathrm{i}}$ for $2 \leqslant \mathrm{i} \leqslant \mathrm{n}$ is doubling on $\mathbb{R}$ and $\omega \in A_{\infty}^{n}$. Let $\left\{R_{k}\right\}$ be a sequence of rectangles in $\mathbb{R}^{n}$ satisfying property $P_{1}$. Then if $M_{\omega \mathrm{d} \mu}^{n}$ is $\mathrm{L}^{\mathrm{p}}(\omega \mathrm{d} \mu)$ bounded with norm at most $\mathrm{O}\left((\mathrm{p}-1)^{-\mathrm{r}}\right), 1<\mathrm{p} \leqslant 2$, for some $\mathrm{r}>0$, one has

$$
\left\|\sum \chi_{R_{k}}\right\|_{L^{p^{\prime}}(\omega \mathrm{d} \mu)} \leqslant C\left(p^{\prime}\right)^{r+1} \omega\left(\cup R_{k}\right)^{1 / p^{\prime}}, \quad 1<p \leqslant 2 .
$$

Proof. Setting $E_{k}=R_{k} \backslash \bigcup_{i<k} R_{i}$, one has $\mu\left(E_{k}\right) \geqslant(1-\epsilon) \mu\left(R_{k}\right)$ by property $P_{1}$ and the fact that the sets $\left\{E_{k}\right\}$ are pairwise disjoint. Since $\omega \in A_{\infty}^{n}(\mu)$, one has $\omega\left(E_{k}\right) \geqslant \beta \omega\left(R_{k}\right)$ for some $0<\beta<1$. Arguing by duality we assume that $\varphi$ is a function satisfying $\|\varphi\|_{L^{p}(\omega \mathrm{d} \mu)}=1, \frac{1}{p}+\frac{1}{p^{\prime}}=1$, then one has

$$
\int \sum \chi_{R_{k}} \varphi \omega d \mu=\sum \int_{R_{k}} \varphi \omega d \mu=\sum\left(\frac{1}{\omega\left(R_{k}\right)} \int_{R_{k}} \varphi \omega d \mu\right) \omega\left(R_{k}\right)
$$




$$
\begin{aligned}
& \lesssim \sum \omega\left(E_{k}\right) \inf _{x \in R_{k}} M_{\omega d \mu}^{n}(\varphi)(x) \\
& \leqslant \int_{\cup E_{k}} M_{\omega d \mu}^{n}(\varphi)(x) \omega(x) d \mu(x) \\
& \leqslant\left\|M_{\omega d \mu}^{n}(\varphi)\right\|_{L^{p}(\omega d \mu)} \omega\left(\cup R_{k}\right)^{1 / p^{\prime}} \\
& \leqslant O\left(\left(p^{\prime}-1\right)^{r}\right) \omega\left(\cup R_{k}\right)^{1 / p^{\prime}} \leqslant O\left(\left(p^{\prime}\right)^{r+1}\right) \omega\left(\cup R_{k}\right)^{1 / p^{\prime}}
\end{aligned}
$$

by the assumption of the $L^{p}(\omega d \mu)$ norm of $M_{\omega d \mu}^{n}$.

Lemma 2.6. Assume that $\mu(x)=\mu_{1}\left(x_{1}\right) \mu_{2}\left(x_{2}\right) \cdots \mu_{n}\left(x_{n}\right)$ is a product measure where $\mu_{i}, i=1, \ldots, n$ are all nonnegative Radon measures in $\mathbb{R}$ without mass-points and complete. Assume also that each $\mu_{i}$ for $2 \leqslant i \leqslant n$ is doubling on $\mathbb{R}$ and $\omega \in A_{\infty}^{n}$. Let $\left\{R_{k}\right\}$ be a sequence of rectangles in $\mathbb{R}^{n}$ satisfying property $P_{2}$. Suppose also that $\mathrm{M}_{\omega^{\prime} \mathrm{d} \mu^{\prime}}^{\mathrm{n}-1}$ is bounded on $\mathrm{L}^{\mathrm{p}}\left(\mathbb{R}^{\mathrm{n}-1}, \omega^{\prime} \mathrm{d} \mu^{\prime}\right)$ with norm at most $\mathrm{O}\left((\mathrm{p}-1)^{-\mathrm{r}}\right), 1<\mathrm{p} \leqslant 2$, uniformly in a.e. $\mathrm{x}_{\mathrm{n}}$ for some $r>0$. Then there exists a constant $C$ independent of $\left\{R_{k}\right\}$ such that

$$
\left\|\sum \chi_{R_{k}}\right\|_{L^{p^{\prime}}(\omega \mathrm{d} \mu)} \leqslant C\left(p^{\prime}\right)^{r+1} \omega\left(\cup R_{k}\right)^{1 / p^{\prime}}, \quad 1<p \leqslant 2 .
$$

Proof. Let $S_{k}^{x_{n}}$ denote the slice of $R_{k}$ by a hyperplane perpendicular to the $x^{n}$-axis, at height $x_{n}$. Using Lemma 2.4, we have

$$
\mu^{\prime}\left(S_{k}^{x_{n}} \cap \bigcup_{i<k} S_{i}^{x_{n}}\right) \leqslant \frac{1}{2} \mu^{\prime}\left(S_{k}^{x_{n}}\right) .
$$

Since $\omega \in A_{\infty}^{n}$, one has $\omega^{\prime} \in A_{\infty}^{n-1}$ uniformly in a.e. $x_{n}$. Then by Lemma 2.5,

$$
\left\|\sum \chi_{S_{k}^{x_{n}}}\right\|_{L_{p^{\prime}}\left(\omega^{\prime} d \mu^{\prime}\right)} \leqslant C\left(p^{\prime}\right)^{r+1} \omega^{\prime}\left(U S_{k}^{x_{n}}\right)^{1 / p^{\prime}}, \quad 1<p \leqslant 2,
$$

uniformly in a.e. $x_{n}$, which follows the desired result by taking the $p^{\prime}$-th power on both sides of above inequality and integrate in $x_{n}$.

Lemma 2.7. Assume that $\mu(x)=\mu_{1}\left(x_{1}\right) \cdot \mu_{2}\left(x_{2}\right) \cdots \mu_{n}\left(x_{n}\right)$ is a product measure where $\mu_{i}, i=1, \ldots, n$ are all nonnegative Radon measures in $\mathbb{R}$ without mass-points and complete. Assume also that each $\mu_{\mathrm{i}}$ for $2 \leqslant i \leqslant n$ is doubling on $\mathbb{R}$ and $\omega \in A_{\infty}^{n}$. Then $M_{\omega \mathrm{d} \mu}^{n}$ is $\mathrm{L}^{\mathrm{p}}(\omega \mathrm{d} \mu)$ bounded with norm at most $\mathrm{O}\left((\mathrm{p}-1)^{-\mathrm{n}}\right), 1<\mathrm{p} \leqslant 2$.

Remark 2.8. The asymptotic estimate of $M^{n}$ was obtained by Long and Shen in [18].

Proof. The proof is by induction on $n$. For $n=1, M_{\omega \mathrm{d} \mu}^{n}$ is the classical Hardy-Littlewood maximal operator $M_{\omega \mathrm{d} \mu}$ with respect to measure $\omega \mathrm{d} \mu$. Let $\lambda>0$ and $E_{\lambda}=\left\{x: M_{\omega \mathrm{d} \mu}(f)>\lambda\right\}$, then the main result of [29] gives

$$
\omega\left(E_{\lambda}\right) \leqslant 5 \lambda^{-1} \int|f(x)| \omega(x) \mathrm{d} \mu(x) .
$$

By interpolation since $M_{\omega \mathrm{d} \mu}$ is $\mathrm{L}^{\infty}(\omega \mathrm{d} \mu)$ to $\mathrm{L}^{\infty}(\omega \mathrm{d} \mu)$ with norm 1 , we obtain

$$
\left\|M_{\omega d \mu}(f)\right\|_{L^{p}(\omega d \mu)} \leqslant c(p-1)^{-1} .
$$

Suppose that $n>1$ and the lemma holds for $n-1$. Since $\omega \in A_{\infty}^{n}$, one has $\omega^{\prime} \in A_{\infty}^{n-1}$ uniformly in a.e. $x_{n}$. By the inductive hypothesis, $M_{\omega^{\prime} d \mu^{\prime}}^{n-1}$ is $L^{p}\left(\omega^{\prime} d \mu^{\prime}\right)$ bounded with norm at most $O\left((p-1)^{1-n}\right)$, $1<\mathrm{p} \leqslant 2$.

Let $\lambda>0$ and and $\left\{R_{k}\right\}$ be a cover of $E_{\lambda}=\left\{x: M_{\omega d \mu}^{n}(f)>\lambda\right\}$ such that

$$
\frac{1}{\omega\left(R_{k}\right)} \int_{R_{k}}|f(x)| \omega(x) d \mu(x)>\lambda .
$$


With no loss of generality, we may assume that $\left\{R_{k}\right\}$ is a finite sequence, and that $R_{k}$ are arranged so that the side length in $x_{n}$ direction is decreasing. We now follow a well-known selecting procedure argument. We choose $R_{1}^{*}=R_{1}$, and assume $R_{1}^{*}, \ldots, R_{k}^{*}$ have been selected. We obtain $R_{k+1}^{*}$ as the first rectangle on the list of $R_{i}$ after $R_{k}^{*}$ such that

$$
\mu\left(R \cap\left[\bigcup_{i \leqslant k} \hat{R}_{i}^{*}\right]\right)<\frac{1}{2} \mu(R) .
$$

That is $\left\{R_{k}^{*}\right\}$ satisfies the property $P_{2}$. By Lemma 2.6, we obtain

$$
\left\|\sum \chi_{R_{k}}\right\|_{L^{p^{\prime}}(\omega \mathrm{d} \mu)} \leqslant \mathrm{C}\left(\mathrm{p}^{\prime}\right)^{\mathrm{n}} \omega\left(\cup \mathrm{R}_{\mathrm{k}}\right)^{1 / \mathrm{p}^{\prime}}, \quad 1<\mathrm{p} \leqslant 2 .
$$

Moreover, arguing as in the proof of Theorem 1.2, one has

$$
\omega\left(\cup R_{k}\right) \lesssim \omega\left(\cup R_{k}^{*}\right)
$$

by the assumption that each $\mu_{i}$ for $2 \leqslant i \leqslant n$ is doubling on $\mathbb{R}$ and $\omega \in A_{\infty}^{n}$. Finally, by (2.1),

$$
\begin{aligned}
\omega\left(\cup R_{k}^{*}\right) \lesssim \sum \omega\left(R_{k}^{*}\right) & \leqslant \sum \frac{1}{\lambda} \int_{R_{k}^{*}}|f(x)| \omega(x) d \mu(x) \\
& \leqslant \frac{1}{\lambda}\left\|\sum \chi_{R_{k}^{*}}\right\|_{L^{p^{\prime}}(\omega \mathrm{d} \mu)}\|f\|_{L^{p}(\omega \mathrm{d} \mu)} \leqslant C\left(p^{\prime}\right)^{n-1} \omega\left(\cup R_{k}^{*}\right)^{1 / p^{\prime}} \frac{1}{\lambda}\|f\|_{L^{p}(\omega d \mu),}
\end{aligned}
$$

from which it follows that

$$
\omega\left(\cup R_{k}^{*}\right) \lesssim\left(C\left(p^{\prime}\right)^{n} \frac{1}{\lambda}\|f\|_{L^{p}(\omega d \mu)}\right)^{p}
$$

Hence, by (2.2),

$$
\omega\left(E_{\lambda}\right) \lesssim\left(C\left(p^{\prime}\right)^{n} \frac{1}{\lambda}\|f\|_{L^{p}(\omega d \mu)}\right)^{p} .
$$

From Lemma 2.3, since $\omega \in A_{p}^{n}(\mu)$, there is an $\varepsilon>0$ such that $\omega \in A_{p-\varepsilon}^{n}(\mu)$. Then (2.3) holds for $p-\varepsilon$. It is also known that $\omega \in A_{p+\varepsilon}^{n}(\mu)$. By interpolation, we complete the proof.

As a direct corollary of Lemma 2.6 and Lemma 2.7, we can obtain the following result.

Corollary 2.9. Assume that $\omega \in A_{\infty}^{n}(\mu)$ and that $\left\{R_{k}\right\}$ is a sequence of rectangles in $\mathbb{R}^{n}$ satisfying property $P_{2}$. Then if $\mathrm{p}$ is big enough,

$$
\left\|\sum \chi_{R_{k}}\right\|_{L^{p}(\omega \mathrm{d} \mu)} \lesssim p^{n} \omega\left(\cup R_{k}\right)^{1 / p}
$$

\section{Endpoint Fefferman-Stein inequality}

Proof of Theorem 1.2. It suffices to prove the theorem for $\lambda=1$. Denote $E=\left\{x: M_{d \mu}^{n}(f)(x)>1\right\}$. Let $\left\{R_{k}\right\}$ be a cover of E such that

$$
\frac{1}{\mu\left(R_{k}\right)} \int_{R_{k}}|f(x)| d \mu(x)>1 .
$$

Since we only need to prove (1.1) for any compact subset $K$ of $E$, without loss of generality, we may assume $\left\{R_{k}\right\}$ is a finite sequence, and $R_{k}$ are arranged so that the side length in $x_{n}$ direction is decreasing.

We now choose a subset $\left\{R_{k}^{*}\right\}$ of $\left\{R_{k}\right\}$ such that $\left\{R_{k}^{*}\right\}$ satisfies property $P_{2}$ and

$$
\omega\left(\cup R_{k}\right) \lesssim \omega\left(\cup R_{k}^{*}\right) .
$$

Let $R_{1}^{*}=R_{1}$, and assume that $R_{1}^{*}, \ldots, R_{k}^{*}$ have been selected. We obtain $R_{k+1}^{*}$ as the first rectangle on the list of $R_{i}$ after $R_{k}^{*}$ such that

$$
\mu\left(R \cap\left[\bigcup_{i \leqslant k} \hat{R}_{i}^{*}\right]\right)<\frac{1}{2} \mu(R) .
$$


This selection process will be end after a finite steps. It is obvious that $\left\{R_{k}^{*}\right\}$ satisfies the property $P_{2}$. Now assume that some $R \in\left\{R_{k}\right\}$ was not selected, then we can find some positive integer $k$ such that

$$
\mu\left(R \cap\left[\bigcup_{i \leqslant k} \hat{R}_{i}^{*}\right]\right) \geqslant \frac{1}{2} \mu(R),
$$

which implies that for all $x \in R$,

$$
M_{\mathrm{d} \mu}^{n}\left(\chi_{i \leqslant k} \hat{\mathrm{R}}_{i}^{*}\right)(x) \geqslant \frac{1}{2}
$$

Hence

$$
\cup R_{k} \subseteq\left\{x: M_{d \mu}^{n}\left(\chi_{\cup \hat{R}_{i}^{*}}\right)(x) \geqslant \frac{1}{2}\right\} .
$$

Since $\omega \in A_{\infty}^{n}$, then $\omega \in A_{p}^{n}(\mu)$ for some $1<p<\infty$. By the result of $M_{d \mu}^{n}$ being bounded on $L^{p}(\omega d \mu)$ ([7, Theorem 1.6]), we conclude that

$$
\omega\left(\cup R_{k}\right) \lesssim \omega\left(\cup \hat{R}_{i}^{*}\right) .
$$

Let $S_{k}^{x_{n}}$ denote the slice of $R_{k}^{*}$ at $x_{n}$ and then $R_{k}^{*}=S_{k}^{x_{n}} \times J_{k}, \hat{R}_{k}^{*}=S_{k}^{x_{n}} \times \hat{J}_{k}$ if $x_{n} \in J_{k}$. Using property $P_{2}$ of $\left\{R_{k}^{*}\right\}$, and Lemma 2.4 we have

$$
\mu^{\prime}\left(S_{k}^{x_{n}} \cap \bigcup_{i<k} S_{i}^{x_{n}}\right) \leqslant \frac{1}{2} \mu^{\prime}\left(S_{k}^{x_{n}}\right)
$$

Since $\omega \in A_{\infty}^{n}$, one has $\omega^{\prime} \in A_{\infty}^{n-1}$ uniformly in a.e. $x_{n}$. Then

$$
\omega^{\prime}\left(S_{k}^{x_{n}} \cap \bigcup_{i<k} S_{i}^{x_{n}}\right) \leqslant \beta \omega^{\prime}\left(S_{k}^{x_{n}}\right)
$$

uniformly in a.e. $x_{n}$, for some $0<\beta<1$. Denote $F_{k}=S_{k}^{x_{n}} \backslash \bigcup_{i<k} S_{i}^{x_{n}}$. It is obvious that $\omega^{\prime}\left(F_{k}\right) \geqslant$ $(1-\beta) \omega^{\prime}\left(S_{k}^{x_{n}}\right)$. By classical result, when $\mu_{n}$ is doubling, $\omega\left(x^{\prime}, x_{n}\right) d \mu_{n}$ is also doubling uniformly for $x^{\prime} \in \mathbb{R}^{n-1}$. Therefore using (3.3)

$$
\begin{aligned}
\omega\left(\cup R_{k}\right) \lesssim \sum \omega\left(\hat{R}_{i}^{*}\right) & =\sum_{k} \int_{S_{k}^{x_{n}}}\left(\int_{\hat{J}_{k}} \omega\left(x^{\prime}, x_{n}\right) d \mu_{n}\left(x_{n}\right)\right) d \mu^{\prime}\left(x^{\prime}\right) \\
& \lesssim \sum_{k} \int_{J_{k}}\left(\int_{F_{k}} \omega\left(x_{1}, x_{2}\right) d \mu^{\prime}\left(x^{\prime}\right)\right) d \mu_{n}=\int_{\cup J_{k} \times F_{k}} \omega(x) d \mu \leqslant \omega\left(\cup_{k} R_{k}^{*}\right),
\end{aligned}
$$

which gives (3.2).

Observe that property $P_{2}$ of $\left\{R_{k}^{*}\right\}$ also implies that

$$
\mu\left(R_{k}^{*} \cap\left[\bigcup_{i<k} R_{i}^{*}\right]\right)<\frac{1}{2} \mu\left(R_{k}^{*}\right) .
$$

It follows that $\omega\left(R_{k}^{*} \cap\left[\bigcup_{i<k} R_{i}^{*}\right]\right) \leqslant \beta \omega\left(R_{k}^{*}\right)$ for some $0<\beta<1$, since $\omega \in A_{\infty}^{n}$. Then setting $E_{k}=$ $R_{k}^{*} \backslash\left[\bigcup_{i<k} R_{i}^{*}\right]$, we have

$$
\omega\left(R_{k}^{*}\right) \geqslant \omega\left(E_{k}\right) \geqslant(1-\beta) \omega\left(R_{k}^{*}\right), \quad \mu\left(R_{k}^{*}\right) \geqslant \mu\left(E_{k}\right) \geqslant \frac{1}{2} \omega\left(R_{k}^{*}\right) .
$$

Using (3.1) and (3.2), we obtain

$$
\omega(E) \lesssim \omega\left(\cup R_{k}^{*}\right) \leqslant \sum \omega\left(R_{k}^{*}\right) \leqslant \sum \frac{\omega\left(R_{k}^{*}\right)}{\mu\left(R_{k}^{*}\right)} \int_{R_{k}^{*}}|f(y)| d \mu(y)=\int|f(y)| \sum_{k} \frac{\omega\left(R_{k}^{*}\right)}{\mu\left(R_{k}^{*}\right)} \chi_{R_{k}^{*}}(y) d \mu(y) .
$$


For locally integrable functions $f$ and $g$, define the linear operators

$$
\operatorname{Tf}(x)=\sum_{k} \frac{1}{\mu\left(R_{k}^{*}\right)} \int_{R_{k}^{*}} f(y) d \mu(y) \chi_{E_{k}}(x), T^{*} f(x)=\sum_{k} \frac{1}{\mu\left(R_{k}^{*}\right)} \int_{E_{k}} f(y) d \mu(y) \chi_{R_{k}^{*}}(x) .
$$

It is easy to check that

$$
\begin{aligned}
\int \mathrm{T} f(x) g(x) d \mu(x) & =\int T^{*} g(x) f(x) d \mu(x), \\
T 1(x) & =\sum_{k} \chi_{E_{k}}(x), \quad T^{*} 1(x)=\sum_{k} \frac{\mu\left(E_{k}\right)}{\mu\left(R_{k}^{*}\right)} \chi_{R_{k}^{*}}(x) \approx \sum_{k} \chi_{R_{k}^{*}}(x),
\end{aligned}
$$

and

$$
\mathrm{T}^{*} \omega(x)=\sum_{k} \frac{\omega\left(\mathrm{E}_{\mathrm{k}}\right)}{\mu\left(\mathrm{R}_{\mathrm{k}}^{*}\right)} \chi_{\mathrm{R}_{\mathrm{k}}^{*}}(\mathrm{x}) \approx \sum_{\mathrm{k}} \frac{\omega\left(\mathrm{R}_{\mathrm{k}}\right)}{\mu\left(\mathrm{R}_{\mathrm{k}}^{*}\right)} \chi_{\mathrm{R}_{\mathrm{k}}^{*}}(\mathrm{x}) .
$$

Hence

$$
\begin{aligned}
\omega\left(\cup R_{k}^{*}\right) \lesssim \int|f(y)| T^{*} \omega(y) d \mu(y) & =\left(\int_{\left\{y: T^{*} \omega(y) \leqslant v(y)\right\}}+\int_{\left\{y: T^{*} \omega(y)>v(y)\right\}}\right)|f(y)| T^{*} \omega(y) d \mu(y) \\
& \leqslant \int f(y) v(y) d \mu(y)+\int_{\left\{y: T^{*} \omega(y)>v(y)\right\}}|f(y)| \frac{T^{*} \omega(y)}{v(y)} v(y) d \mu(y) .
\end{aligned}
$$

Recall a known result from[1]: For any $\theta>0$, there exists a constant $c_{\theta}>0$ such that for all $s, t>0$ we have

$$
s t \leqslant c_{\theta} s\left[1+\left(\log ^{+} s\right)^{n-1}\right]+\exp \left(\theta t^{1 /(n-1)}\right)-1, n \geqslant 2 .
$$

Applying the pointwise estimate above we get for any $\theta>0$,

$$
\begin{aligned}
\int_{\left\{y: T^{*} \omega(y)>v(y)\right\}}|f(y)| \frac{T^{*} \omega(y)}{v(y)} v(y) d \mu(y) \leqslant & c_{\theta} \int_{\left\{y: T^{*} \omega(y)>v(y)\right\}}|f(y)|\left[1+\left(\log ^{+}|f(y)|\right)^{n-1}\right] v(y) d \mu(y) \\
& +\int_{\left\{y: T^{*} \omega(y)>v(y)\right\}}\left(\exp \left(\theta\left(\frac{T^{*} \omega(y)}{v(y)}\right)^{1 /(n-1)}\right)-1\right) v(y) d \mu(y) .
\end{aligned}
$$

Therefore

$$
\omega\left(\cup R_{k}^{*}\right) \lesssim\left(1+c_{\theta}\right) \int|f(y)|\left[1+\left(\log ^{+}|f(y)|\right)^{n-1}\right] v(y) d \mu(y)+I
$$

where

$$
I=\int_{\left\{y: T^{*} \omega(y)>v(y)\right\}}\left(\exp \left(\theta\left(\frac{T^{*} \omega(y)}{v(y)}\right)^{1 /(n-1)}\right)-1\right) v(y) d \mu(y) .
$$

Using the Taylor expansion of $e^{t}$ we can write

$$
I=\sum_{j=1}^{\infty} \frac{\theta^{j}}{j !} \int_{\left\{y: T^{*} \omega(y)>v(y)\right\}}\left(\frac{T^{*} \omega(y)}{v(y)}\right)^{j /(n-1)} v(y) d \mu(y)=\sum_{1 \leqslant j \leqslant n-1}+\sum_{j>n-1}=I_{1}+I_{2} .
$$

For $\mathrm{I}_{1}$, one can easily get

$$
\left(\frac{T^{*} \omega(y)}{v(y)}\right)^{j /(n-1)}=\left(\frac{T^{*} \omega(y)}{v(y)}\right)^{\frac{j}{n-1}-1} \frac{T^{*} \omega(y)}{v(y)} \leqslant \frac{T^{*} \omega(y)}{v(y)},
$$


since $\frac{j}{n-1} \leqslant 1$ and $\frac{T^{*} \omega(y)}{v(y)}>1$. Hence

$$
\mathrm{I}_{1} \leqslant \sum_{1 \leqslant j \leqslant n-1} \frac{\theta^{j}}{j !} \int \theta \mathrm{T}^{*} \omega(y) \mathrm{d} \mu(y) \lesssim_{n} \theta \int \mathrm{T} 1(x) \omega(y) \mathrm{d} \mu(y)=\theta \sum_{k} \int_{E_{k}} \omega(y) d \mu=\theta \omega\left(\cup R_{k}^{*}\right)
$$

from the definition of $T$ and $E_{k}$ provided $\theta<1$.

For item $\mathrm{I}_{2}$, since weights $\omega, v$ satisfy condition $(\mathrm{A})$, one has

$$
\frac{1}{\mu\left(R_{k}^{*}\right)} \omega\left(R_{k}^{*}\right) \leqslant c \inf _{x \in R_{k}^{*}} v(x),
$$

then

$$
\mathrm{T}^{*} \omega(x) \approx \sum_{\mathrm{k}} \frac{\omega\left(\mathrm{R}_{\mathrm{k}}\right)}{\mu\left(\mathrm{R}_{\mathrm{k}}^{*}\right)} \chi_{\mathrm{R}_{\mathrm{k}}^{*}}(\mathrm{x}) \leqslant \sum_{\mathrm{k}} v(x) \chi_{\mathrm{R}_{\mathrm{k}}^{*}}(\mathrm{x}) \approx v(\mathrm{x}) \mathrm{T}^{*} 1(\mathrm{x})
$$

Therefore

$$
\begin{aligned}
I_{2} & =\sum_{j>n-1} \frac{\theta^{j}}{j !} \int\left(\frac{T^{*} \omega(y)}{v(y)}\right)^{j /(n-1)-1} \frac{T^{*} \omega(y)}{v(y)} v(y) d \mu(y) \\
& \lesssim \sum_{j>n-1} \frac{\theta^{j}}{j !} \int\left(T^{*} 1(y)\right)^{j /(n-1)-1} T^{*} \omega(y) d \mu(y) \\
& \lesssim \sum_{j>n-1} \frac{\theta^{j}}{j !} \int\left(T^{*} 1(y)\right)^{j /(n-1)} T^{*} \omega(y) d \mu(y) \\
& =\sum_{j>n-1} \frac{\theta^{j}}{j !} \int_{\cup R_{k}^{*}} T\left(T^{*} 1(y)\right)^{j /(n-1)} \omega(y) d \mu(y) .
\end{aligned}
$$

Observing that $\operatorname{Tf}(x) \leqslant \sum_{k} \chi_{E_{k}}(x) \inf _{y \in R_{k}^{*}} M_{d \mu}^{n}(f)(y) \leqslant M_{d \mu}^{n}(f)(x)$, so we have

$$
\|\mathrm{Tf}\|_{\mathrm{L}^{\mathrm{p}_{0}}(\omega \mathrm{d} \mu)} \lesssim\|f\|_{\mathrm{L}^{\mathrm{p}_{0}}(\omega \mathrm{d} \mu)}
$$

for some $1<p_{0}<\infty$, since $\omega \in A_{\infty}^{n}$. This together with Corollary 2.9 and Hölder's inequality yield

$$
\begin{aligned}
\int_{\cup R_{k}^{*}} T\left(T^{*} 1(y)\right)^{j /(n-1)} \omega(y) d \mu(y) & \lesssim \omega\left(\cup R_{k}^{*}\right)^{\frac{1}{p_{0}^{\prime}}}\left\|\left(T^{*} 1\right)^{j /(n-1)}\right\|_{L^{p_{0}}(\omega d \mu)} \\
& =\omega\left(\cup R_{k}^{*}\right)^{\frac{1}{p_{0}^{\prime}}}\left(\int\left(T^{*} 1\right)^{j p_{0} /(n-1)} \omega(y) d \mu(y)\right)^{\frac{1}{p_{0}}} \lesssim\left(\frac{j p_{0}}{n-1}\right)^{j} \omega\left(\cup R_{k}^{*}\right),
\end{aligned}
$$

which follows that

$$
I_{2} \lesssim \sum_{j>n-1} \frac{\theta^{j}}{j !}\left(\frac{j p_{0}}{n-1}\right)^{j} \omega\left(\cup R_{k}^{*}\right) \lesssim n \sum_{j>n-1} \frac{\left(e \theta p_{0} /(n-1)\right)^{j}}{\sqrt{j}} \omega\left(\cup R_{k}^{*}\right) \lesssim n \frac{\left(e \theta p_{0} /(n-1)\right)^{n}}{\sqrt{n}} \omega\left(\cup R_{k}^{*}\right),
$$

by choosing $\theta$ small enough such that $e \theta p_{0} /(n-1)<1$.

At last, we obtain that

$$
\omega\left(\cup R_{k}^{*}\right) \lesssim \omega, n\left(1+c_{\theta}\right) \int|f(y)|\left[1+\left(\log ^{+}|f(y)|\right)^{n-1}\right] v(y) d \mu(y)+\left(\theta+\frac{\left(e \theta p_{0} /(n-1)\right)^{n}}{\sqrt{n}}\right) \omega\left(\cup R_{k}^{*}\right),
$$

which yields that

$$
\omega\left(\cup R_{k}^{*}\right) \lesssim \int|f(y)|\left[1+\left(\log ^{+}|f(y)|\right)^{n-1}\right] v(y) d \mu(y),
$$

by letting $\theta$ sufficiently small.

Thus we complete the proof using (3.2). 


\section{Acknowledgment}

This work is supported by the National Natural Science Foundation of China (No. 11501308, No. 11771223).

\section{References}

[1] R. J. Bagby, Maximal functions and rearrangements: some new proofs, Indiana Univ. Math. J., 32 (1983), 879-891. 1, 3

[2] A. Chang, R. Fefferman, Some recent developments in Fourier Analysis and $\mathrm{H}^{\mathrm{p}}$ theory on product domains, Bull. Amer. Math. Soc., 12 (1985), 1-43. 1

[3] A. Cordoba, Maximal functions, covering lemmas and Fourier multipliers, In: Harmonic Analysis in Euclidean Spaces (Proc. Sympos. Pure Math.), 1979 (1979), 29-50. 1

[4] A. Cordoba, R. Fefferman, A geometric proof of the strong maximal theorem, Ann. Math. Second Series, 102 (1975), 95-100.

[5] A. Cordoba, C. Fefferman, A weighted norm inequality for singular integrals, Studia Math., 57 (1976), 97-101. 1

[6] D. Cruz-Uribe, J. M. Martell, C. Pérez, Sharp two weight inequalities for singular integrals, with applications to the Hilbert transform and the Sarason conjecture, Adv. Math., 216 (2007), 647-676. 1

[7] W. Ding, L. X. Jiang, Y. P. Zhu, Boundedness of strong maximal functions with respect to non-doubling measures, J. Inequal. Appl., 2016 (2016), 14 pages. 1, 1, 2, 2, 3

[8] R. Fefferman, Some weighted norm inequalities for Cordoba's maximal function, Amer. J. Math., 106 (1984), $1261-1264$. 1

[9] R. Fefferman, Strong differentiation with respect to measures, Amer. J. Math., 103 (1981), 33-40. 1

[10] R. Fefferman, J. Pipher, Multiparameter operators and sharp weighted inequalites, Amer. J. Math., 119 (1997), $337-369$. 1

[11] C. Fefferman, E. Stein, Some maximal inequalities, Amer. J. Math., 93 (1971), 107-115. 1

[12] J. Garcia-Cuerva, J. L. Rubio de Francia, Weighted norm inequalities and related topics, North-Holland Publishing Co., Amsterdam, (1985).

[13] L. Grafakos, Classical Fourier Analysis, Springer-Verlag, New York, (2008). 1

[14] Y. Han, M. Y. Lee, C. C. Lin, Y. C. Lin, Calderón-Zygmund operators on product Hardy spaces, J. Fourier Anal., 258 (2010), 2834-2861. 1

[15] Y. S. Han, C. C. Lin, G. Z. Lu, Z. P. Ruan, E. T. Sawyer, Hardy spaces associated with different homogeneities and boundedness of composition operators, Rev. Mat. Iberoam., 29 (2013), 1127-1157. 1

[16] B. Jawerth, A. Torchinsky, The strong maximal function with respect to measures, Studia Math., 80 (1984), $261-285.1$

[17] J. L. Journé, Calderman-Zygmund operators on product spaces, Rev. Mat. Iberoam., 1 (1985), 55-92. 1

[18] R. L. Long, Z. W. Shen, A note on a covering lemma of A. Cordoba and R. Fefferman, Chinese Ann. Math. Ser. B, 9 (1988), 283-291. 2.8

[19] T. Luque, I. Parissis, The endpoint Fefferman-Stein inequality for the strong maximal function, J. Fourier Anal., 266 (2014), 199-212. 1, 1

[20] J. Mateu, P. Mattila, A. Nicolau, J. Orobitg, BMO for nondoubling measures, Duke Math. J., 102 (2000), 533-565. 1

[21] T. Mitsis, The weighted weak type inequality for the strong maximal function, J. Fourier Anal. Appl., 12 (2006), 645-652. 1

[22] B. Muckenhoupt, E. M. Stein, Classical expansions and their relation to conjugate harmonic functions, Trans. Amer. Math. Soc., 118 (1965), 17-92. 1

[23] F. Nazarov, S. Treil, A. Volberg, Cauchy integral and Calderón-Zygmund operators on nonhomogeneous spaces, Internat. Math. Res. Notices, 15 (1997), 703-726. 1

[24] F. Nazarov, S. Treil, A. Volberg, The Bellman function and two weight inequalities for Haar multipliers, J. Amer. Math. Soc., 12 (1999), 909-928. 1

[25] J. Orobitg, C. Pérez, $A_{p}$ weights for nondoubling measures in $\mathrm{R}^{\mathrm{n}}$ and applications, Trans. Amer. Math. Soc., 354 (2002), 2013-2033. 1

[26] C. Perez, A remark on weighted inequalities for general maximal operators, Proc. Amer. Math. Soc., 119 (1993), 11211126. 1

[27] C. Perez, Weighted norm inequalities for general maximal operators, Publ. Mat., 35 (1991), 169-186. 1

[28] E. T. Sawyer, A characterization of a two weight norm inequality for maximal operators, Studia Math., 75 (1982), 1-11. 1

[29] P. Sjögren, A remark on maximal function for measures in $\mathrm{R}^{\mathrm{n}}$, Amer. J. Math., 105 (1983), 1231-1233. 2

[30] E. M. Stein, Harmonic analysis: real-variable methods, orthogonality, and oscillatory integrals, Princeton University Press, Princeton, (1993). 1

[31] X. Tolsa, BMO, $\mathrm{H}^{1}$ and Calderón-Zygmund operators for non doubling measures, Math. Ann., 319 (2001), 89-149. 1 